\title{
On Justice Knowledge Activation: Evidence for Spontaneous Activation of Social Justice Inferences
}

\author{
Jaap Ham $\cdot$ Kees van den Bos
}

Published online: 1 February 2011

(C) The Author(s) 2011. This article is published with open access at Springerlink.com

\begin{abstract}
This article focuses on how people infer the justness of events they encounter. Earlier justice research typically asked participants explicitly for their justice judgments. More recent research provided evidence for the possibility of spontaneous judgment inferences. The present research extends this study in three important ways: it provides strong evidence that (1) spontaneous social justice inferences can occur in multiple research paradigms, (2) these inferences constitute a process separate from spontaneous general evaluation of valence, and (3) spontaneous justice inferences covary with individual differences in sensitivity to justice. We provide evidence for these three conclusions by means of important implicit measurement research paradigms that we specifically tailored to study justice inferences: the probe recognition paradigm and the grid-relearning paradigm. We discuss the implications of our findings for both the literatures on justice and spontaneous inferences.
\end{abstract}

Keywords Social justice - Spontaneous activation · Inferences · Judgments · Probe recognition - Grid relearning

Social justice represents a core issue in society, politics, organizations, intimate relationships-indeed in almost every social situation in which people interact with each other (Folger, 1984). Study in various scientific disciplines (ranging from economics to ethology; see, e.g., Beauchamp, 2001) suggests that in their day-to-day

J. Ham · K. van den Bos

Department of Social and Organizational Psychology, Utrecht University, Utrecht, The Netherlands

J. Ham $(\bowtie)$

Department of Human-Technology Interaction, Eindhoven University of Technology, IPO 1.36, P.O. Box 513, 5600 MB Eindhoven, The Netherlands

e-mail: j.r.c.ham@tue.nl 
lives people are exposed to justice-related issues repeatedly. Because all these encounters inherently demand that people assess the justice of what was going on (as justice is not an innate property of a situation but rather in the eye of the beholder; Mikula \& Wenzel, 2000), assessing what is just and unjust may be assumed crucial and frequent human activities. Indeed, recent research provides preliminary evidence that individuals spontaneously judge the justness or unjustness of events that they encounter (Ham \& Van den Bos, 2008; see also, Correia, Vala, \& Aguitar, 2007; Hafer, 2000; Kay \& Jost, 2003). That is, there is some tentative evidence suggesting that people can draw social justice inferences without having the intention to form an impression of whether the event that happened was just or unjust (for a more extensive introduction to the concept of spontaneous inferences, see Uleman, 1999; Uleman, Adil Saribay, \& Gonzalez, 2008).

We think it is important to build and extend on these research studies indicating that people can infer justice levels of situations spontaneously because more systematic evidence for the spontaneous qualities of the justice judgment process is needed. Part of why we think more systematic evidence is needed is conceptual. That is, earlier studies have disputed that individuals consider the justice implications of events spontaneously (see, e.g., Jasso, 1999; Sabbagh, Dar, \& Resh, 1994). Related to this, well-known moral psychologists have stated explicitly that assessing morality is caused primarily by careful, intentional reasoning processes (e.g., Kohlberg, 1969; Piaget, 1975; Turiel, 1983) whereas others assume, yet do not show, that assessing morality "is generally the result of quick, automatic evaluations (intuitions)" (Haidt, 2001, p. 814).

Another reason why we argue more systematic evidence for spontaneous justice inferences is needed is empirical. For example, most earlier justice studies did not assess the spontaneity of people's judgments of the justness or unjustness of events and did not use spontaneous justice judgments as their main dependent variables. That is, almost all studies reported in the literature explicitly asked participants or respondents to form justice judgments using explicit measures like Likert-type rating scales to assess justice ratings (for an overview, see, e.g., the appendix in Lind \& Tyler, 1988; see also Colquitt \& Shaw, 2005). By definition, these kinds of judgments are intentional (Uleman, 1999) and therefore spontaneously formed justice judgments have largely not been assessed (notwithstanding that they may have been activated).

There have been earlier articles that, although they did not directly assess the spontaneity of justice judgments, have argued that after many social experiences people may have justice on their minds (see, e.g., Greenberg, 1986, see also, Lerner $\&$ Goldberg, 1999). Also, further support for our argument for spontaneous justice judgments can be found in recent studies that suggest that spontaneous activation of justice-related knowledge indeed may occur (Correia et al., 2007; Hafer, 2000; Kay \& Jost, 2003). For example, Hafer (2000) found that participants' implicit reactions to justice-related words were influenced when participants' beliefs in a just world were threatened. However, these studies (Correia et al., 2007; Hafer, 2000; Kay \& Jost, 2003) do not provide direct evidence for spontaneous, unintentional activation of justice-related knowledge: justice judgments may have been intentional in these studies, but they do assess implicit responses related to the activation of the concept of justice. 
More recently there has been direct evidence reporting that people may spontaneously draw inferences about social justice when they encounter events that may or may not strongly threaten their just-world beliefs (Ham \& Van den Bos, 2008). That is, in two studies we investigated the social justice inferences people draw after reading descriptions that are highly personally relevant (e.g., "You and your colleague do the same work. You make 1400 euros a month and your colleague makes 4100 euros a month") versus after reading descriptions of lower personal relevance (e.g., "He and his colleague do the same work. He makes 1400 Euros a month and his colleague makes 4100 Euros a month"). In both studies, we not only assessed intentional inferences about social justice (using explicit Likert-type justice judgment scales), but also assessed spontaneous justice inferences (using an implicit measurement paradigm: the probe recognition paradigm). Results indicated that participants drew inferences about justice spontaneously. Furthermore, the personal relevance manipulation did not affect intentional inferences about social justice, but did influence spontaneous justice inferences. That is, spontaneous justice inferences were stronger following the reading of descriptions of high personal relevance than descriptions of lower personal relevance. Building on this insight we will present self-relevant descriptions to our participants in the current experiments, noting that spontaneous justice inferences are possible also using descriptions that are less selfrelevant.

\section{The Current Research}

Our 2008 findings were the first direct evidence for spontaneous justice inferences using an implicit research paradigm that is well-suited to assess spontaneous inferences (Uleman, Hon, Roman, \& Moskowitz, 1996) and provide preliminary evidence for the idea that people spontaneously draw inferences about social justice (Ham \& Van den Bos, 2008). The present research extends on the earlier research in three important ways: a first aim of the current research is to provide more robust evidence that spontaneous social justice inferences can occur, using multiple research paradigms. That is, we will provide evidence from the probe recognition paradigm that we used in our earlier research (Ham \& Van den Bos, 2008), and from a new research paradigm as well (the grid-relearning paradigm). Obtaining evidence from multiple paradigms is important before reliable conclusions about spontaneous inferences can be drawn (see, e.g., Uleman, 1999).

A second aim of the current research is to provide evidence that spontaneous social justice inferences constitute a process separate from spontaneous general evaluation of valence. We think it is necessary to test this prediction, because a critic might argue that the spontaneity of justice inferences simply constitutes a process of merely evaluating the positivity or negativity of events as opposed to more specific spontaneous evaluation of justice inferences. Thus, it is important to show a difference in spontaneous justice inferences as compared to spontaneous valence evaluation (Fazio, 2001). The second aim of this article, therefore, is to test the specificity of the spontaneity of justice inferences versus more general spontaneous valence evaluation. 
A third aim is to show that spontaneous justice inferences can covary with individual differences in sensitivity to justice. Research suggests that people differ in the strength with which they react to just and unjust events. These individual differences can be measured by means of the justice sensitivity scale (JSS) developed by Schmitt (1996; Schmitt, Gollwitzer, Maes, \& Arbach, 2005). This scale consists of three subscales, of which the first subscale $\left(\mathrm{JSS}_{\text {Victim }}\right)$ measures sensitivity with regard to experiencing injustice towards oneself. The second subscale (JSS Perpetrator ) measures sensitivity to profiting oneself from unjust events.

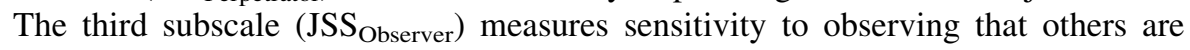
treated unjustly. We argue here that the more sensitive people are to issues of social justice, the more they will spontaneously draw inferences about social justice. From a functional perspective, it can very well be stated that functionally it would make the most sense if people high in sensitivity to issues of social justice would be able to form adequate justice judgments most spontaneously. Therefore, in line with earlier findings from the social-cognitive literature that higher mental processes that occur often tend to become automatized (Smith, 1994; see also Smith \& Lerner, 1986), we propose that people who are strongly sensitive to justice issues may have made justice judgments more frequently, and, thereby, are more likely to draw spontaneous justice inferences than people who are less strongly sensitive to justice issues. Earlier research did not test this prediction, and we think it is important to show evidence for this hypothesis because it will lead to a better view on the mechanisms leading to spontaneous justice inferences.

\section{Experiment 1}

In social cognition research, the automaticity of higher mental processes has been studied extensively. Findings show, for instance, that after reading the behavior description "John gets an A for the test," the trait inference "smart" might be spontaneously activated (e.g., Ham \& Vonk, 2003). In addition to the reasons mentioned earlier, we think it is interesting to investigate the spontaneity of social justice inferences because we would like to try to integrate the social cognition and social justice literatures; domains of social psychology that have been developed in largely independent ways yet that may profit from each other's insights. Building on the literature on spontaneous trait inferences, therefore, we used implicit measures to find evidence for spontaneous justice inferences. Specifically, we used two important different implicit measurement paradigms to find evidence for spontaneous justice inferences: probe recognition and grid-relearning paradigms (Uleman, Newman, \& Moskowitz, 1996). In both the experiments of this article, we presented participants with descriptions that implied a just event or an unjust event had happened as well as with descriptions that did not imply anything related to justice. After this, we measured spontaneous justice inferences using these implicit measurement paradigms.

In Experiment 1, we assessed participants' spontaneous justice inferences by means of the probe recognition paradigm (McKoon \& Ratcliff, 1986). In this research paradigm, participants read a short description of a situation after which a 
probe word was presented on their screens. Participants were asked to accurately indicate as quickly as possible whether this probe word was part of the sentence they read. For example, participants in our experimental trials read the sentence "You and your colleague do the same work. You make 1400 Euros a month, and you colleague 4100 Euros," while on control trials they read a sentence that used the same words (including identical pronouns to refer to the actor) but which did not imply anything related to social justice. After such a sentence had been presented, the word "just" was presented on participants' computer screen and participants were asked to indicate whether this probe word was part of the sentence they had read. If justice inferences are spontaneously drawn while reading the experimental description, then we should find that participants need more time to respond and/or more often respond incorrectly on experimental trials as compared to control trials (the probe recognition effect). This is because the spontaneous justice inference might have interfered with correctly and quickly rejecting the probe word after reading a description that did imply that probe word more so than after reading a description that did not (McKoon \& Ratcliff, 1986). Therefore, finding the probe recognition effect indicates that participants spontaneously drew justice inferences. Additionally, in the probe recognition paradigm, participants must suppress drawing spontaneous inferences to respond quickly and correctly as instructed. Therefore, this paradigm provides strong evidence that inferring the probe concepts is not only unintended but also difficult to control (McKoon \& Ratcliff, 1986).

What makes the probe recognition paradigm especially suited to study spontaneous justice inferences is that, by its very nature, it pits ostensible task demands against spontaneous inferences (Uleman, Hon, et al., 1996). That is, to respond quickly and correctly as instructed, participants must suppress drawing inferences about social justice. Thereby, this paradigm provides strong evidence that a justice inference was formed without intentions to do so, and in efficient, and, especially, uncontrolled ways (see Bargh, 1994). Additionally, characteristics of the probe recognition paradigm are such that awareness of the formation of a justice inference is avoided (McKoon \& Ratcliff, 1986; Uleman, Newman, et al., 1996). That is, one of the important characteristics of the probe recognition paradigm is that of all the descriptions presented to participants in this paradigm, justice-related descriptions need only be a small minority. More specifically, our participants are only presented justice-related descriptions on 4 of all 60 descriptions, and awareness of the formation of a justice inference is thereby hidden between interpreting an abundance of other (filler) descriptions. In sum, the probe recognition paradigm can provide strong evidence that spontaneous justice inferences can be formed during reading of situation descriptions that portray a just or an unjust event.

More specifically, the probe recognition paradigm uses short description as stimulus material to guarantee implicit measurement and spontaneity of cognitive activity (see McKoon \& Ratcliff, 1986). Therefore, we developed and pre-tested four different, short descriptions of justice-implying situations. Of all four descriptions, we developed three versions; a just-implying version and an unjustimplying version (e.g., a description of a just [unjust] event was: "On the first class, the professor states that 2 [1] of 2 assignments must be handed in. After the course, both assignments are used to calculate your grade"); and a control version. All three 
versions used identical words. The just-implying and the unjust-implying versions solely differed in numbers or amounts mentioned (e.g., "2" or "1") such that they either implied just or unjust events. Some of the four experimental descriptions involved distributive justice, some procedural justice. The control version presented the words (of either the just-implying or the unjust-implying version) in a different order such that these control descriptions formed logical sentences that did not imply anything related to justice (e.g., "On the first class, the professor states assignment calculations used in both 2 grades. After the course, the 2 [1] assignments must be handed in.").

All descriptions were self-involving because earlier research (Ham \& Van den Bos, 2008) suggests that highly self-relevant justice-related situation descriptions cause stronger spontaneous justice inferences than low self-relevant descriptions. A pretest (Pretest 1, described in Footnote 1) indicated that for all four different descriptions, the three versions indeed were perceived to be just, unjust, and neutral on a dimension of unjust to just. ${ }^{1}$

Our predictions were that we would find effects on the probe recognition measures indicating that participants draw justice inferences spontaneously when they read the descriptions that implied just or unjust events. Furthermore, we used our implicit measurement paradigms also to assess spontaneous evaluations of general valence, and, thus, to explore the possible differences between spontaneous justice inferences and spontaneous valence evaluation.

\section{Method}

\section{Participants and Design}

Participants were 89 students (25 men and 64 women) at Utrecht University. All participants were native Dutch speakers. For a participation of $20 \mathrm{~min}$ they received 2 Euros (1 Euro equaled approximately $\$ 1.20$ U.S. at the time the studies in this paper were conducted). All participants were submitted to a 2 (trial type: experimental vs. control) $\times 2$ (description: just-implying vs. unjust-implying) $\times$ 2 (probe type: justice-related vs. valence-related) within participants design.

\footnotetext{
1 In Pretest 1, 160 participants were asked to evaluate how just $(1=$ very unjust, $7=$ very just $)$, fair ( 1 = very unfair, 7 = very fair), appropriate $(1=$ very inappropriate, $7=$ very appropriate $)$, and justified ( 1 = very unjustified, $7=$ very justified) they thought the events described in the sentences to be. For each sentence, participant's answers to these four items were averaged to form a reliable scale of their justice judgments (for each sentence, alpha $>.84$ ). Results indicated that events described in justimplying descriptions were indeed judged to be more just (on average, $M=6.3, S D=1.4$ ) than unjustimplying descriptions (on average, $M=1.5, S D=0.9$ ), $p \mathrm{~s}<.001$, for all four descriptions. Also, the control version of each sentence was judged $(M=4.3, S D=1.2)$ to be more just than the unjustimplying version, $p s<.001$, for all four descriptions, and less just than the just-implying descriptions, $p s<.001$, for all four descriptions. Furthermore, an analysis of response times to these questions did not show a difference in response times influenced by description type, $F<1$. Therefore, Pretest 1 shows that, as intended, that processing times of the three types of descriptions are comparable.
} 


\section{Stimulus Materials}

In our probe recognition paradigm, participants completed 60 trials in random order. In each trial, a short description and a single probe word were presented to the participants. Of the 60 trials, 8 trials ( 4 experimental and 4 control trials) were the critical trials that were used to test our hypotheses.

In the 4 experimental trials, each description was succeeded by a probe word that was implied by the description. Two experimental trials each employed a description implying just events, whereas the remaining 2 experimental trials each employed a description implying unjust events. In both these 2 sets of 2 experimental trials, the probe word was a justice-related word in one trial, and a valence-related word in the other trial.

The 4 control trials were similar to the 4 experimental trials, but used descriptions that presented the words (of either the just-implying or the unjustimplying version) in a different order such that these control descriptions formed logical sentences that did not imply anything related to social justice. All descriptions are available on request. So, to keep words used in the control descriptions completely the same as in the experimental descriptions, we used control descriptions in two versions: either the control description used completely the same words as the just-implying experimental description or completely the same words as the unjust-implying experimental description. For the 2 experimental trials employing a justice-related probe word and the corresponding control trials, 2 probe words were taken randomly from a set of 8 words related to social justice. As just probe words we used "just," "fair," "justified," and "appropriate," and as unjust probe words we used "unjust," "unfair," "unjustified," and "inappropriate." 2 Using both just and unjust probe words allowed us to assess differences in activation levels between these two types of justice-related probes.

For the 2 experimental trials employing a valence-related probe word and the corresponding control trials, 2 probe words were taken randomly from a set of 4 words related to general valence evaluation. The positive probe words "positive" and "friendly" represented positive evaluation, and the negative probe words "negative" and "hateful". The valence of all probe words was assessed in Pretest 2. ${ }^{3}$ For all trials, at random a description and a concurring probe were chosen with this restriction that no 2 trials used the same materials.

\footnotetext{
${ }^{2}$ All stimulus materials presented are the English translations of the Dutch materials we used.

${ }^{3}$ In Pretest 2, implicit general valence evaluations of all cue words used in Experiments 1 and 2 were assessed using a measure of implicit evaluation (the Extrinsic Affective Simon Task or EAST; see De Houwer, 2003). In the EAST, participants see white words that need to be classified on the basis of stimulus valence and colored words that need to be classified on the basis of color. One can estimate participants' general valence evaluation of a stimulus by presenting that stimulus on the colored trials and comparing the time needed to give the extrinsically positive response (i.e., the response that has to be made to positive white words) with the time needed to give an extrinsically negative response (i.e., the response that has to be made to negative white words). If on the colored trials, the extrinsically positive response is given more quickly and with fewer errors than the extrinsically negative response, one can infer that the stimulus presented on those trials is positive. If the reverse is true, then the stimulus is evaluated negatively.
} 
In addition to the 8 critical trials, 52 filler trials unrelated to social justice were included in the experiment for two reasons. First, if all materials were justicerelated, we would not be able to show that it is specifically spontaneity of justice inferences (as opposed to other, more general inferences) that we are revealing here (McKoon \& Ratcliff, 1986). Second, if all correct responses were to answer "no," attending to the description would not be necessary to complete the task (McKoon $\&$ Ratcliff, 1986). Therefore, in 30 of the 52 filler trials the probe was a word that was literally in the description. Filler descriptions and probe words were comparable in length and sentence structure to the critical descriptions and probe words.

\section{Experimental Procedure}

The experiment was run on a computer and started with an explanation of the first task. The first task was the probe recognition task which started with a practice round of 12 trials. The practice rounds were the same as the trials of the experimental task, but materials were unrelated to social justice. Next, the 60 trials of the main task started. In each trial, first a row of X's appeared in the middle of the screen for $1000 \mathrm{~ms}$. Next, the description was displayed for $3000 \mathrm{~ms}$, followed by a blank screen lasting $500 \mathrm{~ms}$. Again a row of X's appeared on screen for $500 \mathrm{~ms}$, this time followed by the probe. During the whole task the words "yes" and "no" remained visible on the right and left side of the screen. From the moment the probe was presented, participants could press the appropriate keys (the "a"-key and the "6"-key to indicate "no" and "yes," respectively) to give their answer. The probe

\section{Footnote 3 continued}

Specifically, the 40 participants of Pretest 2 completed an EAST that used 4 sets of words: 2 sets of valence-related words ( 2 positive and 2 negative) and the justice-related words ( 2 just-related and 2 unjust-related) of Experiments 1 and 2. Within participants, we manipulated set contents (justice-related or valence-related words) and set valence (positive or negative). For each set, we calculated an EAST response time score and an EAST error rate score, identical to De Houwer (2003, Study 2). Positive EAST scores indicate that the stimulus presented on those trials is evaluated positive, whereas negative EAST scores indicate negative valence evaluation. We analyzed EAST response time scores and EAST error rate scores (as De Houwer, 2003) for the 4 sets of cue words in two separate 2 (set contents: justicerelated vs. valence-related) x 2 (set valence: positive vs. negative) repeated measures ANOVA's. In both analyses, we found the expected interaction effect; for EAST response time scores, $F(1,39)=4.85$, $p<.05$; for EAST error rate scores, $F(1,39)=5.00, p<.05$. As expected, inspection of means reveals that for valence-related cue words differences in EAST scores between positive and negative sets are bigger than for justice-related cue words. That is, the interaction effect indicated that for EAST response time scores, valence-related positive set scores $(M=40 \mathrm{~ms}, S D=55 \mathrm{~ms})$ differed more from valencerelated negative set scores $(M=-37 \mathrm{~ms}, S D=51 \mathrm{~ms}), F(1,39)=45.83, p<.001, \eta^{2}=.54$, than justice-related positive set scores $(M=16 \mathrm{~ms}, S D=54 \mathrm{~ms})$ differed from justice-related negative set scores $(M=-24 \mathrm{~ms}, S D=50 \mathrm{~ms}), F(1,39)=10.25, p<.01, \eta^{2}=.21$. Similarly, for EAST error rate scores the above mentioned interaction effect showed that the difference between valence-related positive and negative error rate scores $(M \mathrm{~s}=1.17 \%$ vs. $-0.98 \%, S D \mathrm{~s}=.95$ and $.76 \%)$ was bigger, $F(1$, $39)=104.36, p<.001, \eta^{2}=.73$, than the difference between justice-related positive and negative error rate scores $(M \mathrm{~s}=.81 \%$ vs. $-0.73 \%, S D \mathrm{~s}=1.03$ and $.89 \%), F(1,39)=62.39, p<.001, \eta^{2}=.61$. These findings show that the cue words representing valence evaluation are evaluated more extreme on a scale of valence than are words representing justice. As intended, this made our valence-related words better suited for assessing automatic general evaluation than our justice-related words (cf. De Houwer, 2003), because, as expected, these results show that they lead to stronger implicit evaluations. 
remained visible until the participant had pressed one of both keys. After the answer was given, a blank screen was displayed for $1000 \mathrm{~ms}$. Then, the next trial started.

After completing all trials, participants completed the most recent version of the justice sensitivity scales (Schmitt et al., 2005). For each subscale, participants had to indicate on a 7-point Likert scale how much they agreed with 10 different statements $(1=$ totally disagree, $7=$ totally agree $)$. Participants' answers to each set of 10 questions were averaged to form three reliable scales of JSS $_{\text {Victim }}$ $($ alpha $=.78), \quad \mathrm{JSS}_{\text {Observer }} \quad($ alpha $=.89)$, and $\mathrm{JSS}_{\text {Perpetrator }} \quad($ alpha $=.86)$. An example of a $\mathrm{JSS}_{\text {Victim }}$ statement is "It bothers me when others receive something that ought to be mine." An example of a $\mathrm{JSS}_{\text {Observer }}$ statement is "I am upset when someone does not get a reward he/she has earned." And an example of a $\mathrm{JSS}_{\text {Perpetrator }}$ statement is "I cannot easily bear to unilaterally profit from others." Finally, participants were thanked, paid for their participation and debriefed.

\section{Results}

\section{Response Latencies}

All participants had a low error rate $(M=5.7 \%$, ranging from 0 to $11.2 \%)$. Response latencies were analyzed only if the reaction had been a correct one. As recommended by Ratcliff (1993), we analyzed our response latency data by using two methods. First, an absolute cutoff criterion of $<200$ and $>2000$ ms was used. Second, an inverse transformation $(1 / x)$ of the response latencies was used. The analyses reported hereafter are based on the cutoff criterion, which yielded converging results to the inverse transformation analysis. By using the cutoff point criterion, only a total of 7 responses $(0.16 \%)$ from 5 participants had to be dropped from the statistical analyses.

Response latencies were submitted to a 2 (trial type: experimental vs. control) $\times$ 2 (description: just-implying vs. unjust-implying) $\times 2$ (probe type: justice-related vs. valence-related) repeated measures analysis of variance (ANOVA). We found corroborative evidence for our research hypotheses. That is, we found evidence for spontaneous justice inferences, indicated by an interaction between trial type and probe type, $F(1,83)=5.67, p<.05$. As predicted, for justice-related probe words, participants responded slower on experimental trials $(M=699, S D=174)$ than on control trials $(M=639, S D=204), F(1,83)=13.15, p<.01$. For valence-related probe words, participants did not respond slower on experimental trials than on control trials (overall $M=651, S D=215$ ),$F<1$. So, as expected, participants were slower in rejecting justice-related probe words on experimental trials (after reading a description that implied just or unjust events) than on a control trials (after reading a justice-unrelated control description), whereas for valence-related probe words this effect was weaker, in fact was statistically not significant. Furthermore, there was no statistical evidence that descriptions implying just events led to different probe recognition effects than descriptions implying unjust events, as indicated by a non-significant interaction between trial type and description, $F<1$, and between trial type, description, and probe type, $F<1$. 
We also examined whether the 4 just probe words led to different probe recognition effects than the 4 unjust probe words. Because we only allowed for a minimum number of 2 experimental trials to contain only materials (description and probe word) related to justice, each participant saw only two of the four possible combinations of the two types of descriptions (just-implying or unjust-implying) and the two types of justice-related probe words (just and unjust probe words). Because of randomization of these combinations, there were two specific subsets of participants that did see the trials relevant for the current analyses. That is, one subset saw only just probe words and a sentence implying a just event on the one and an unjust event on the other of the two experimental trials. Another subset saw only unjust probe words combined with both a sentence implying a just event and one implying an unjust event. So, we could perform two separate 2 (trial type: experimental vs. control) $\times 2$ (description: just-implying vs. unjust-implying) repeated measures ANOVAs for the two subsets of participants. Results indicate probe recognition effects for both types of probe words: For just probe words, participants responded slower on experimental trials $(M=667, S D=181)$ than on control trials $(M=618, S D=195), F(1,20)=4.44, p<.05$, and for unjust probe words, this pattern was also found $(M=689$, and $S D=134$, versus $M=599$, and $S D=168), F(1,20)=7.37, p<.05$, independent of whether the sentence implied just or unjust events, all $F \mathrm{~s}<1$.

Likewise, we performed two separate ANOVAs identical to those used to analyze response times to justice-related probe words, now to explore whether the positive valence-related probe words led to different response times than negative valencerelated probe words. Results indicate no overall probe recognition effects for both types of probe words: Neither for positive nor for negative valence-related probe words did we find a main effect of trial type, both $F_{\mathrm{s}}<1$. However, we did find that descriptions that implied a just event led to spontaneous activation of positive valence evaluation. That is, for positive valence-related probe words, we found an interaction between trial type and description: Simple effect analyses revealed that when the description implied a just event, participants responded slower to positive valence-related probe words on experimental trials $(M=739, S D=263)$ than on control trials $(M=635, S D=177), F(1,20)=4.73, p<.05$, but not when then the description implied an unjust event, $F<1$. For negative valence-related probewords, this interaction was not found, $F<1$.

\section{Error rates}

Because no selection criteria apply in this analysis, all error rates of the 89 participants could be analyzed in repeated measures ANOVA identical to the one used to analyze response latencies. ${ }^{4}$ This analysis showed results comparable to the analysis of response latencies: The interaction effect between trial type and probe type was significant, $F(1,88)=17.98, p<.001$. For justice-related probes, participants made more errors on experimental trials $(M=6.49 \%, S D=0.58 \%)$

\footnotetext{
4 When analyzing error rates, we will analyze binomial data-i.e., answers were either correct or wrong. For analyses of variance on binomial data, see Kirk (1982).
} 
than on control trials $(M=4.04 \%, S D=0.57 \%), F(1,88)=33.94, p<.001$. For probes related to evaluation, this difference in error rates was not found (overall $M=5.59 \%, S D=0.60 \%), F<1$. Furthermore, as in the analyses of participants' response times, there was no evidence that descriptions implying just events led to different error rates than did descriptions implying unjust events, as suggested by a non-significant interaction between trial type and description, $F<1$, and between trial type, description, and probe type, $F<1$.

Finally, comparable to the response time results, further analyses of error rates for just versus unjust probe words indicated probe recognition effects for both just as well as unjust probe words. Two separate repeated measures ANOVA's, identical to those used to analyze response times of just and unjust probe words, showed that participants made more errors on experimental trials than on control trials, both for just-related probe words, $F(1,22)=6.52, p<.05$, as well as for unjust-related probe words, $F(1,21)=8.89, p<.01$, independent of description type, all $F \mathrm{~s}<1$.

We also performed identical ANOVAs to assess probe recognition effects on error rates for positive and negative valence-related probe words. Comparable to analyses of response times on the two types of valence-related probe words, results did not show that participants made more errors on experimental trials than on control trials for positive nor for valence-related probe words. However, different from analyses on response times to positive valence-related probe words, the analyses of error rates did not suggest that descriptions that implied a just event led to activation of spontaneous positive valence evaluation. That is, for positive evaluation-related probe words, we did not find an interaction between trial type and description in the analysis of error rates, $F(1,22)=1.53, p=.23$, and a simple main effect of trial type was not found for descriptions implying a just event nor descriptions implying an unjust event, $F_{\mathrm{s}}<1$. For negative valence-related probe words, comparable to the analyses of response times, differences in error rates between descriptions types were not found, $F<1$.

\section{Insights from Differences in Justice Sensitivity}

An additional, intriguing aspect of the current findings is that they suggest that spontaneous justice inferences and individual differences in justice sensitivity may be correlated. Specifically, we analyzed whether participants' scores on the three subscales of the justice sensitivity scale (JSS) were related to the spontaneous activation of the justice-related probe words. To this end, we constructed a measure representing the spontaneous activation of justice-related probe words by subtracting participants' response times on control trials from their response times on experimental trials (collapsing across just-implying and unjust-implying descriptions). A regression analysis on this measure for spontaneous justice inferences,

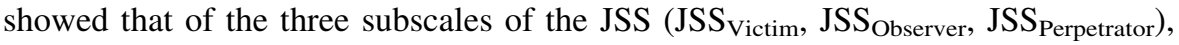
$\mathrm{JSS}_{\text {Victim }}$, Beta $=.236, t(85)=1.96, p<.05$, and $\mathrm{JSS}_{\text {Perpetrator }}$, Beta $=.238$, $t(85)=2.12, p<.05$, were significantly related to participants' spontaneous justice inferences. This suggests that especially individual differences in sensitivity to experiences of own injustice and sensitivity towards profiting from unfair events may be strongly associated with spontaneous justice inferences. 


\section{Discussion}

In accordance with our expectations, Experiment 1 presents evidence for spontaneous justice inferences. That is, when participants had read a justiceimplying description and the probe word was justice-related, participants were slower and made more errors on rejecting the probe. This suggests that a justice inference was drawn spontaneously while reading our experimental descriptions and that this spontaneous justice inference interfered with quickly and correctly rejecting the probe. Because the probe recognition paradigm was designed to guarantee spontaneity of activation of inferences (see, Uleman, 1999), and hence not giving participants any intentions or instructions to start forming justice judgments), the findings of Experiment 1 indicate that people can activate concerns for social justice in unintended ways when witnessing justice-related events.

The findings of Experiment 1 also indicate that spontaneously drawing an inference about social justice is not necessarily the same as more general, spontaneous processes of valence evaluation, as the findings obtained in our probe recognition paradigm clearly indicated stronger activation by our justice-implying descriptions for justice-related probe words than for valence-related probe words. That is, the results indicate only spontaneous justice inferences, but, overall, no spontaneous activation of valence evaluation. Importantly, the results did suggest that the probe recognition paradigm we used in Experiment 1 is sensitive to detecting spontaneous valence evaluation. That is, when our participants had read a description that implied a just event, participants were slower on rejecting positive valence-related probe, although they did not make more errors. This suggests that positive valence has been spontaneously activated while reading those descriptions as it interfered with quickly rejecting the probe. This indicates that some spontaneous valence evaluation has occurred. In Experiment 2, we will use another implicit measurement paradigm to study the robustness of the effects reported in Experiment 1.

Finally, we also obtained evidence that individual differences in sensitivity to experiences of own injustice and sensitivity toward profiting from unfair events may be strongly associated with spontaneous justice inferences. We did not find differences in activation of just-related from unjust-related concepts dependent on whether a description implied a just or an unjust event. We will come back to these findings after we have reported the findings of our second experiment.

\section{Experiment 2}

One important aim of Experiment 2 was to replicate the findings obtained in Experiment 1 using a different research paradigm. To this end, we adapted another implicit measurement paradigm to detect spontaneous justice inferences: the "gridrelearning paradigm." This paradigm was recently developed by Ham and Vonk (2003) to study the activation of spontaneous inferences in person perception. It is in general based on the notion that relearning is more effective than learning, and more specifically on research by Carlston and Skowronski (1994), and on the idea that 
implicit inferences are learned when they initially occur, so that when the same material is encountered later on in explicit learning trials, it is essentially relearned and hence learned more quickly.

In the grid-relearning paradigm, we developed for the line of research presented here, participants had the opportunity to learn a combination two times. That is, in the three tasks of this paradigm, participants are presented with a $4 \times 4$ information-grid. In the first task, in each cell of the grid, a description is presented. For example, one unjust-implying description that we used was: "You and your colleague do the same work. You make 1400 euros a month and your colleague makes 4100 euros a month." This description was presented to the participants in a certain cell of the $4 \times 4$ grid and the only instruction participants received was to read this sentence. In the second task, cue words are presented in each cell and participants are asked to remember which word was presented in which cell. Finally, in the third task, recall for which word was presented in which cell is tested.

In some cases (labeled a relearning trial), the cue word presented in the second task is an implication of the description presented in that same cell in the previous task. For example, "unjust" is presented in the same cell as where the unjustimplying description was presented. In such a case, assuming that a justice inference is already drawn spontaneously while reading the description during the first task (since no instructions were given to participants to form justice judgments), ${ }^{5}$ this implies that participants are now learning a combination they already saw before. In effect, then, they are relearning the combination. In other cases (labeled control trials), the cue word presented in the second task is not an implication of the description presented in that same cell in the first task. For example, "unjust" is presented in the same cell as where a description that did not imply something related to justice was presented in the first task. So, in the control trials, relearning does not occur because a spontaneous justice inference cannot have been drawn while reading this description.

If a justice inference is drawn spontaneously while reading the justice-implying description in the first task, recall should be better in relearning trials than in control trials, because in case of the relearning trial, the first exposure should have produced a residual effect that facilitates learning in the second task. These facilitation effects (Carlston \& Skowronski, 1994; Ham \& Vonk, 2003), were used here to study the possibility of spontaneous justice inferences in Experiment 2.

\footnotetext{
5 Indeed, not giving instructions to form a justice inference does not necessarily mean that people did not intentionally form one. However, as the relearning paradigm does give participants another instruction (namely to read the sentences presented to them), this research paradigm (see also Carlston \& Skowronski, 1994) argues that they were not given the intention to form justice inferences but rather other intentions, and therefore, that participants (if the relearning effect is found) drew justice inferences spontaneously, that is, without haven been given the intention. People may spontaneously come up with the intention to draw justice inferences (instead of doing what we ask them: read the sentences), but in that case, their intentions would be at least spontaneously formed. Experiment 1 indicates that even when participants need to suppress drawing spontaneous inferences (to respond quickly and correctly as instructed) they still do so. Thereby, the research paradigm used in Experiment 1 indicates that even when something like 'spontaneous intentions to draw justice inferences' is inhibited, we find evidence of spontaneous justice inferences.
} 
The relearning paradigm is especially suited for our current means because it has not only been used to assess spontaneous social inferences, but also to assess spontaneous valence evaluation. That is, in a study by Ho, Skowronski, and Carlston (D. Carlston, personal communication, November 11, 2005), the relearning paradigm showed facilitation effects for general valence evaluation terms like "positive" and "negative," indicating that valence evaluation spontaneously occurred when participants were presented with positive or negative valenceimplying (behavior) descriptions, and can be detected by relearning paradigms. Because the experimental descriptions we used in Experiment 2 were specifically tailored to reflect justice-related issues, we expected participants who have been presented just-implying and unjust-implying descriptions to show stronger facilitation effects for cue words that are related to justice than for cue words related to general valence evaluation, and exploratively we examined whether facilitation effects on general valence evaluation would be found in our justice-related relearning paradigm.

Method

\section{Participants and Design}

Ninety-four students (33 men and 61 women) at Utrecht University participated in this experiment. All participants were native Dutch speakers and received 2 Euros for their participation of approximately $20 \mathrm{~min}$. Participants were submitted to a 3 (trial type: relearning just-implying description vs. relearning unjust-implying description vs. control) $\times 2$ (cue type: justice-related word vs. valence-related word) within participants design.

\section{Stimulus Materials}

In the experiment, each participant completed 16 trials. Ten of these trials were filler trials and 6 were experimental trials. In each trial, a short description and a single cue word were presented to the participants. In the 6 experimental trials, each participant was presented with 2 just-implying descriptions, 2 unjust-implying descriptions, and 2 justice-unrelated descriptions. The descriptions were presented in a randomized order to participants. The just-implying and unjust-implying descriptions used in Experiment 2 are identical to those used in Experiment 1. The justice-unrelated descriptions were 2 general personality-trait-implying descriptions, taken at random from a set of 12 descriptions used in earlier research using this paradigm (see Ham \& Vonk, 2003, Appendix, Descriptions 1-12). Finally, on the 10 filler trials, the remaining 10 descriptions from the Ham and Vonk (2003) materials were used.

The cue words used on the experimental trials of Experiment 2 were identical to the justice-related and valence-related probe words used in Experiment 1. Like the descriptions, cue words used on the 10 filler trials were identical to those used in earlier research using this paradigm (see Ham \& Vonk, 2003, Appendix, Trait words 1-10). For each experimental trial, at random a description and a cue word were chosen with this restriction that no two trials used the same materials. 


\section{Overview of the Grid-Relearning Paradigm}

The grid-relearning paradigm consisted of three main tasks: an exposure task, a relearning task, and a cued-recall task. In each task, a grid of $4 \times 4$ cells was displayed on participants' screens. In the exposure task, 16 descriptions were presented: One by one, in random order and randomly distributed among the cells of the grid, a description was displayed for $6 \mathrm{~s}$. In the relearning task, a randomly ordered cue word was displayed for $4 \mathrm{~s}$ in one of the cells. Participants were instructed to memorize which word was displayed in which cell. In half of the trials - the relearning trials - the cue word fitted an implication of the description that had been presented in the same cell during the exposure task. In the other half of the trials - the control trials - the displayed cue word did not fit an implication of the description presented in the same cell. Thus, these control trials did not allow relearning. Finally, during the cued-recall task, participants were asked to recall what cue word was shown in which cell in the relearning task.

So, the grid-relearning paradigm consists of 16 trials, of which 8 were relearning trials and 8 were control trials. Of all 16 trials, 3 trials ( 2 of the 8 relearning trials and 1 of the 8 control trials) were used to detect spontaneous justice inferences and the other 3 trials ( 2 of the remaining 6 relearning trials and 1 of the remaining 7 control trials) were used to detect spontaneous evaluation of valence. The design of the current study only applies to these 6 trials. The remaining 10 trials ( 4 relearning trials and 6 control trials) were filler trials that did not use material related to justice. As in Experiment 1, the filler trials were included mainly to keep the ratio of justicerelated descriptions to justice-unrelated descriptions low. Cue type was manipulated within the six experimental trials: In 3 trials, the cue word used in the relearning task was a justice-related word, and in the other 3 trials a valence-related word. Also within these 6 experimental trials, thus within subjects, trial type was manipulated. That is, participants completed two relearning trials that used a just-implying description, two relearning trials that used an unjust-implying description and two control trials. In the two types of relearning trials, the description was just-implying in the one type of relearning trials, and the description was unjust-implying in the other. In control trials, the descriptions in the exposure task did not imply anything related to just or unjust experiences.

\section{Procedure}

Seated behind a computer screen, participants individually went through the instructions and the tasks. They were told that they would participate in a study investigating communication. After receiving general instructions, participants completed a practice trial, consisting of completing a practice exposure task and a practice cued-recall task (both tasks were unrelated to social justice).

After this, the actual experiment began, starting with the exposure task. Participants were asked to read all descriptions to be presented in the $4 \times 4$ grid. In each cell of the grid, in random order, a description was presented for $6 \mathrm{~s}$. After a description had been presented, the screen turned blank for $2 \mathrm{~s}$ and then the next description was displayed in another cell. 
Between the exposure and the relearning task, a filler task (consisting of five word puzzles) was inserted to make it less easy for participants to recall the specific information presented in each cell (cf. Carlston \& Skowronski, 1994). After the filler task, participants completed the relearning task. Identical to the practice task, participants were asked to remember what cue word was presented in which cell in this task. Each cue word was presented for $2 \mathrm{~s}$ in a cell of the grid, and then the screen turned blank for $2 \mathrm{~s}$ and the next word was presented in another cell. Between the relearning and the cued-recall task, a second filler task (consisting of a different set of five word puzzles) was inserted.

The last task was the cued-recall task. In this task, participants were presented with the words presented in the relearning task, and asked in which cell that cue word had been shown during the relearning task. This question was asked about all 16 words, in random order. Every time, the question was presented underneath the $4 \times 4$ grid, and participants could answer by clicking on a cell with their mouse. So, the dependent variable indicated whether the participant answered a question correct or false (coded as " 1 " for a correct answer and " 2 " for an incorrect answer on a specific trial).

After the grid-relearning paradigm, participants completed the justice sensitivity scales, identical to Experiment 1. As in Experiment 1, participants' answers to each set of 10 questions were averaged to form the three reliable scales of JSS $_{\text {Victim }}$ $($ alpha $=.81), \mathrm{JSS}_{\text {Observer }}($ alpha $=.86)$, and $\mathrm{JSS}_{\text {Perpetrator }}($ alpha $=.82)$. Finally, participants were debriefed and thanked for their participation.

Results and Discussion

\section{Facilitation Effects}

To determine whether we found evidence for spontaneous justice inferences in Experiment 2, we analyzed whether the justice relearning paradigm we developed yielded the facilitation effects predicted by our focal hypothesis of Experiment 2. Therefore, whether participants' responses to the cued-recall on a trial were correct or incorrect was submitted to a 3 (trial type: relearning just-implying description vs. relearning unjust-implying description vs. control) $\times 2$ (cue type: justice-related word vs. valence-related word) repeated measures ANOVA (see Footnote 3). A difference in activation of spontaneous justice inferences as compared to spontaneous valence evaluation would be indicated by facilitation effects on justice-related cue words for relearning trials over control trials. In other words, we expected an interaction between trial type and cue type such that superior recall would be found on the relearning trials for justice-related cue words. This interaction effect between trial type and cue type was indeed found, $F(1,93)=3.15, p<.05$. As hypothesized, for justice-related cue words, relearning trials led to better recall of cue words than the control trials, indicated by a simple effect of trial type, $F(1,93)=4.26, p<.05$. That is, a facilitation effect was found for justice-related cue words: Of all participants, $47.3 \%$ remembered the relearning trials correctly, whereas $30.9 \%$ remembered the control trial correctly, indicated by a contrast comparing (the two types of) relearning trials to control trials, $F(1,93)=10,03, p<.001$. 
Results of Experiment 2 also mirror those of Experiment 1 by showing that whether the descriptions implied just or unjust events did not influence facilitation effects on recall of justice-related cue words: Contrast analyses showed that the facilitation effect was found both for relearning trials using a just-implying description (in which case $48.9 \%$ of the participants answered correctly) compared to control trials $(30.9 \%$ of the participants answered correctly), $F(1,93)=7.96$, $p<.01$, as for relearning trials using an unjust-implying description (45.7\% of the participants answered correctly) compared to control trials, $F(1,93)=5.72$, $p<.05$. A direct comparison of these two effects suggests that just-implying versus unjust-implying sentences did not lead to better relearning of justice-related cue words, as indicated by a non-significant contrast effect, $F<1$.

For valence-related cue words, a facilitation effect following the just- or unjustimplying descriptions was not found: Relearning trials were not remembered better than control trials for these cue words, $F<1$ (overall, $44.3 \%$ of the participants answered correctly on these trials). ${ }^{6}$ Also, both the contrast of relearning trials using a just-implying description versus control trials and the contrast of relearning trials using an unjust-implying description to control trials were not significant, and also a contrast comparing (the two types of) relearning trials to control trials was not significant, all $F \mathrm{~s}<1$.

Furthermore, we explored whether the 4 just cue words led to different facilitation effects than the 4 unjust cue words. Replicating the pattern found in Experiment 1, results of Experiment 2 indicate a facilitation effect for both types of cue words: For just cue words, $57.9 \%$ of the participants remembered relearning trials correctly, whereas only $34.2 \%$ remembered the control trial correctly, $F(1$, $37)=6.13, p<.05$, and for unjust cue words, this pattern was also found $(58.1-24.3 \%$ of the participants), $F(1,37)=11.24, p<.01$, independent of whether the description implied just or unjust events, $F \mathrm{~s}<1$.

We also explored whether the 2 positive valence-related cue words led to different facilitation effects than the 2 negative valence-related cue words. Although these analyses in Experiment 1 did reveal some evidence of specific spontaneous valence-evaluations, results of the current experiment did not indicate facilitation effects, neither when the probe word was related to positive or negative valence nor when the description implied a just or an unjust event, $F \mathrm{~s}<1$.

\section{Insights from Differences in Justice Sensitivity}

Additionally, Experiment 2 corroborates the findings obtained in Experiment 1 in that spontaneous justice inferences and the same individual differences in justice sensitivity $\left(\mathrm{JSS}_{\text {Victim }}, \mathrm{JSS}_{\text {Perpetrator }}\right.$ ) were again significantly related: As in Experiment 1, we analyzed whether participants' scores on the justice sensitivity scale (JSS) subscales were related to a measure we constructed that indicated the strength of spontaneous activation of justice-related cue words. This indication of the

\footnotetext{
${ }^{6}$ A direct comparison between findings for justice-related cue words and valence-related cue words cannot be made because these types of cue words consist of different words that can be more easy or more difficult to remember.
} 
strength of spontaneous justice inferences was calculated by subtracting scores on control trials from scores on experimental trials for trials using justice-related cue words. A regression analysis with all three subscales of the JSS as predictors, and this measure for spontaneous justice inferences as the criterion, again showed $\mathrm{JSS}_{\text {Perpetrator }}$, Beta $=.34, t(90)=2.61, \quad p<.05$, and $\mathrm{JSS}_{\text {Victim }}$, Beta $=.25$, $t(90)=2.27, p<.05$, to be significant predictors, whereas $\mathrm{JSS}_{\text {Observer }}$ was not a significant predictor, Beta $=.032, t(90)=.25, p=.80$.

\section{General Discussion}

The current findings support and extend earlier research that provided the first evidence for spontaneous justice inferences (Ham \& Van den Bos, 2008). That is, the current research provides further evidence that people can draw inferences about the justness of situations spontaneously, using the identical research paradigm to assess spontaneous justice inferences as earlier research (Ham \& Van den Bos, 2008; the probe recognition paradigm) but also using a new research paradigm (the grid-relearning paradigm). More specifically, in two experiments, using the two different implicit measurement paradigms, we found evidence that people spontaneously draw inferences about the justness of events. In Experiments 1 and 2 , we found evidence that people draw spontaneous inferences about social justice when reading a self-relevant situation description of a just or an unjust event. That is, we found that when people read short situation descriptions that portray just or unjust events, they spontaneously activate social justice inferences, resulting in stronger interference effects in a probe-recognition study (Experiment 1) and stronger facilitation effects in a relearning study (Experiment 2). Because of the features of the currently used research paradigms described earlier (for further details, see Carlston \& Skowronski 1994; McKoon \& Ratcliff, 1986; see also De Houwer \& Moors, 2007), we know that spontaneous justice inferences studied here have been formed in unintentional (indicated by both research paradigms) and uncontrollable (indicated by the probe recognition paradigm, see also, Bargh, 1994) ways by our participants, and we can assume that the justice inferences drawn were activated spontaneously while reading the descriptions (see Uleman, 1999). The current results provide further evidence that in many justice-relevant situations people will draw inferences about justice levels of that situation without the necessity of any intentions to assess justice levels, and without being able to refrain from doing so. Therefore, this finding presents empirical evidence to the ongoing debate on the nature of justice and moral judgments mentioned in the introduction (see, e.g., Haidt, 2001): justice judgments do not necessarily need careful and intentional reasoning, but can also be made unintentionally. An implication of the current findings therefore is that it can be expected that people will spontaneously draw inferences about social justice in justice-relevant situations, also when they are busy doing other things (e.g., Van den Bos, Peters, Bobocel, \& Ybema, 2006).

Furthermore, the results presented here suggest that spontaneous justice inferences should not be equated with spontaneous general evaluation of valence. That is, both experiments clearly indicated stronger spontaneous justice inferences 
than spontaneous general valence evaluation, indicated by stronger interference effects for justice-related words than for valence-related words in Experiment 1, and stronger facilitation effects for justice-related words than for valence-related words in Experiment 2. Thus, both the experiments show that justice-implying descriptions influenced activation of justice-related words stronger than activation of valencerelated words. Had spontaneous justice inferences been a process of more general valence evaluation, we should have found no significant differences between justice-related and valence-related probes or cues, or would have found the opposite pattern of results, because, as Pretest 2 indicates, our valence-related words are evaluated more extreme on a valence dimension than the justice-related words and both research paradigms are sensitive to detecting both spontaneous justice inferences as well as spontaneous valence evaluation. ${ }^{7}$ This last aspect of our findings suggests that, on the one hand, as Van den Bos et al. (2008) suggest, the cognitive process of assessing social justice seems to be a process identical in its characteristics to other higher mental processes, but, on the other hand, it also seems to constitute an independent higher mental processes that can have unique qualities, at least in the two experiments presented here.

Another major aim of the current manuscript was to assess the relation between our measures of spontaneous justice judgments and sensitivity to issues of social justice. In Experiments 1 and 2, we found that sensitivity for social justice issues (Schmitt, 1996) predicted the strength of spontaneous justice inferences. More precisely, the subscales of $\mathrm{JSS}_{\text {Victim }}$ and $\mathrm{JSS}_{\text {Perpetrator }}$ predicted the strength of spontaneous justice inferences, whereas the subscale of $\mathrm{JSS}_{\text {Observer }}$ did not. This indicates that the more sensitive people are to experiencing injustice towards oneself $\left(\mathrm{JSS}_{\text {Victim }}\right)$ and to profiting themselves from unfair events $\left(\mathrm{JSS}_{\text {Perpetrator }}\right)$, the more strongly they spontaneously drew justice inferences in our studies. Contrastingly, spontaneous justice inferences remained independent of sensitivity to observing that

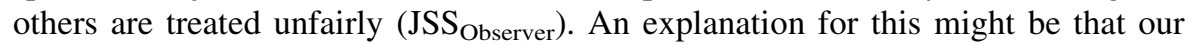
stimulus material only used descriptions of justice-relevant situations applied to participants themselves (as, e.g., "You made a test of 40 questions. Your grade is based on 1 of your 40 answers") and did not use descriptions of justice-relevant situations applied to other people. So, interestingly, this suggests that spontaneous justice inferences about self-involving justice-related situations can be predicted by specific sensitivity to issues of social justice, namely sensitivity to self-involving issues ( $\mathrm{JSS}_{\text {Victim }}$ and $\mathrm{JSS}_{\text {Perpetrator }}$ ), and not by sensitivity to other-involving issues of social justice. Future research may want investigate whether spontaneous justice inferences about other-involving justice-related situations can be predicted by sensitivity to other-involving issues of social justice (the $\mathrm{JSS}_{\text {Observer }}$ subscale). The current findings corroborate our argument for the idea that frequently assessing (self-involving) social justice (reflected in high levels of justice sensitivity) will lead to more spontaneous (self-involving) justice inferences. Thereby, these findings are

\footnotetext{
7 That is, Experiments 1 and 2 indicate that both research paradigms are sensitive to detection of spontaneous justice inferences. In addition, as mentioned earlier, findings of Experiment 1 suggest that the probe recognition paradigm is sensitive to detecting spontaneous valence evaluation, and earlier research (by Ho, Skowronski, \& Carlston; D. Carlston, personal communication, November 11, 2005) successfully used the relearning paradigm of Experiment 2 to detect spontaneous valence evaluation.
} 
relevant to recent work by Baumert and Schmitt (2009) that frames justice sensitivity in terms of the accessibility of justice categories. So, in Experiments 1 and 2 we found evidence for spontaneous inferences about social justice, but no evidence that unjust-implying descriptions lead to stronger automatic activation of justice concerns than just-implying descriptions.

Furthermore, the current two studies did also not suggest differences in activation of just-related (e.g., "just") from unjust-related concepts (e.g., "unjust") dependent on whether a description implied a just or an unjust event. This latter finding is in line with the finding that negations of evaluative stimuli (e.g., "no snakes") do not influence spontaneous evaluative responses to these stimuli (Deutsch, Gawronski, \& Strack, 2006; but see also Mayo, Schul \& Burnstein, 2004), even though spontaneous responses to stimuli that consist of traits versus their antonyms (e.g., "cruel" vs. "kind") can vary (Todorov \& Uleman, 2002). A measure of concept activation (as the two research paradigms we used in Experiments 1 and 2) might be very sensitive to activation levels of concepts (represented by words or related words), but might not be the best paradigm to assess activation differences between concepts represented by words related to one another. Future research may employ implicit measurement paradigms that are better suited for these specific questions, and in addition study possible mediators of the strength of spontaneous justice inferences.

Recently, Uleman et al. (2008) indicated that spontaneous inferences have been insufficiently linked to other phenomena and theories. The current research makes this link in three ways: By integrating the social cognition (including spontaneous inference literature) and social justice literatures, disentangling spontaneous inferences from spontaneous general evaluations, and assessing the covariance between spontaneous justice inferences and sensitivity to justice. Thereby, it adds important knowledge to the literature on spontaneous inferences.

A final merit of the current research we want to mention is that it provides methodological tools that can be used in future research to get more fine-grained insights into the social justice judgment process. That is, we have tailored two precise research paradigms and ditto stimulus materials especially to assess and test spontaneous justice inferences.

More broadly, the current findings have important implications for proponents of models in moral psychology and social justice research that assume either controlled or spontaneous cognitive processes for making justice judgments or moral judgments. Because we now have strong and additional evidence that justice judgments can occur spontaneously, we can conclude that not necessarily are controlled processes needed for people to come to justice judgments. Therefore, the current results suggest a disconfirmation of an important assumption of earlier justice models stating that the justice judgment process constitutes a particularly controlled process (see, e.g., Jasso, 1999; Sabbagh et al., 1994). Rather, we can conclude that cognitive reactions to justice-related events probably consist of both spontaneous (as indicated by the current research) and controlled (as indicated by earlier research) processes (see, e.g., Strack \& Deutsch, 2004), and future research could investigate the relation between these processes. This could lead to the conclusion that, whether 
born-in (De Waal, 2001) or through socialization (see, e.g., Lerner, Miller, \& Holmes, 1976), humans seem to be able to make justice judgments spontaneously.

In sum, the current research teaches us several things about justice inferences: it provides strong evidence that spontaneous social justice inferences can occur in multiple research paradigms, these inferences constitute a process separate from spontaneous general evaluation of valence, and spontaneous justice inferences covary with individual differences in sensitivity to justice. Also, we now have available research mechanisms to directly assess these spontaneous justice inferences.

Thereby, the current research sheds light on the nature of justice inferences. It suggests that the nature of people's responses to justice-relevant situations and events might at least partly be spontaneous and unintentional. So, when assessing people's justice-relevant responses, future research should take into account people's spontaneous justice inferences. Because reflective cognitions (e.g., controlled justice judgments) and implicit cognitions (e.g., spontaneous justice inferences) relate to behavior differently (Strack \& Deutsch, 2004), spontaneous justice inferences might prove to be important determinants of behavior in crucial situations. For example, recent research indicates that implicit attitudes about consumer products are good product choice predictors in situations of high timeconstraint (Friese, Waenke, \& Plessner, 2006). Future research might investigate the relationship between controlled and spontaneous justice inferences and various kinds of justice-related behavior. Thereby, we would not only uncover more about the nature of justice inferences, but also about the implications of the (spontaneous, implicit) nature of justice inferences on human behavior in society, politics, organizations, and intimate relationships.

Acknowledgments The research reported in this article was supported by a VICI innovational research grant from the Netherlands Organization for Scientific Research (NWO, 453.03.603) awarded to Kees van den Bos. We thank Annemarie Loseman for providing the Dutch translation of the justice sensitivity scale.

Open Access This article is distributed under the terms of the Creative Commons Attribution Noncommercial License which permits any noncommercial use, distribution, and reproduction in any medium, provided the original author(s) and source are credited.

\section{References}

Bargh, J. A. (1994). The four horsemen of automaticity: Awareness, efficiency, intention, and control in social cognition. In R. S. Wyer Jr. \& T. K. Srull (Eds.), Handbook of social cognition (2nd ed., pp. 1-40). Hillsdale, NJ: Erlbaum.

Baumert, A., \& Schmitt, M. (2009). Justice-sensitive interpretations of ambiguous situations. Australian Journal of Psychology, 61, 6-12.

Beauchamp, T. L. (2001). Philosophical ethics: An introduction to moral philosophy (3rd ed.). Boston: McGraw-Hill.

Carlston, D. E., \& Skowronski, J. J. (1994). Savings in the relearning of trait information as evidence for spontaneous inference generation. Journal of Personality and Social Psychology, 66, 840-856. 
Colquitt, J. A., \& Shaw, J. C. (2005). How should organizational justice be measured? In J. Greenberg \& J. A. Colquitt (Eds.), Handbook of organizational justice (pp. 113-152). Mahwah, NJ: Lawrence Erlbaum Associates.

Correia, I., Vala, J., \& Aguitar, P. (2007). Victim's innocence, social categorization, and the threat to the belief in a just world. Journal of Experimental Social Psychology, 43, 31-38.

De Houwer, J. (2003). The extrinsic affective Simon task. Experimental Psychology, 50, 77-85.

De Houwer, J., \& Moors, A. (2007). How to define and examine the implicitness of implicit measures. In B. Wittenbrink \& N. Schwarz (Eds.), Implicit measures of attitudes (pp. 179-194). New York: Guilford Press.

De Waal, F. (2001). The ape and the sushi master: Cultural reflections of a primatologist. New York: Basic Books.

Deutsch, R., Gawronski, B., \& Strack, F. (2006). At the boundaries of automaticity: Negation as reflective operation. Journal of Personality and Social Psychology, 91, 386-405.

Fazio, R. H. (2001). On the automatic activation of associated evaluations: An overview. Cognition and Emotion, 15, 115-141.

Folger, R. (Ed.). (1984). The sense of injustice: Social psychological perspectives. New York: Plenum.

Friese, M., Waenke, M., \& Plessner, H. (2006). Implicit consumer preferences and their influence on product choice. Psychology \& Marketing, 23, 727-740.

Greenberg, J. (1986). Determinants of perceived fairness of performance evaluations. Journal of Applied Psychology, 71, 340-342.

Hafer, C. L. (2000). Do innocent victims threaten the belief in a just world? Evidence from a modified Stroop task. Journal of Personality and Social Psychology, 79, 165-173.

Haidt, J. (2001). The emotional dog and its rational tail: A social intuitionist approach to moral judgment. Psychological Review, 108, 814-834.

Ham, J., \& Van den Bos, K. (2008). Not fair for me! The influence of personal relevance on social justice inferences. Journal of Experimental Social Psychology, 44, 699-705.

Ham, J., \& Vonk, R. (2003). Smart and easy: Co-occurring activation of spontaneous trait inferences and spontaneous situational inferences. Journal of Experimental Social Psychology, 39, 434-447.

Jasso, G. (1999). How much injustice is there in the world? Two new justice indexes. American Sociological Review, 64, 133-168.

Kay, A. C., \& Jost, J. T. (2003). Complementary justice: Effects of "poor but happy" and "poor but honest" stereotype exemplars on system justification and implicit activation of the justice motive. Journal of Personality and Social Psychology, 85, 823-837.

Kirk, R. E. (1982). Experimental design: Procedures for the behavioral sciences. Belmont, CA: Brooks/ Cole.

Kohlberg, L. (1969). Stage and sequence: The cognitive-developmental approach to socialization. In D. A. Goslin (Ed.), Handbook of socialization theory and research (pp. 347-480). Chicago: Rand McNally.

Lerner, M. J., \& Goldberg, J. H. (1999). When do decent people blame victims? The differing effects of the explicit/rational and implicit/experiential cognitive systems. In S. Chaiken \& Y. Trope (Eds.), Dual-process theories in social psychology (pp. 627-640). New York: Guilford Press.

Lerner, M. J., Miller, D. T., \& Holmes, J. G. (1976). Deserving and the emergence of forms of justice. In L. Berkowitz \& E. Walster (Eds.), Advances in experimental social psychology (Vol. 9, pp. 133-162). New York: Academic Press.

Lind, E. A., \& Tyler, T. R. (1988). The social psychology of procedural justice. New York: Plenum.

Mayo, R., Schul, Y., \& Burnstein, E. (2004). "I am not guilty" vs "I am innocent": Successful negation may depend on the schema used for its encoding. Journal of Experiment Social Psychology, 40, 433-449.

McKoon, G., \& Ratcliff, R. (1986). Inferences about predictable events. Journal of Experimental Psychology. Learning, Memory, and Cognition, 12, 82-91.

Mikula, G., \& Wenzel, M. (2000). Justice and social conflict. International Journal of Psychology, 35, $126-135$.

Piaget, J. (1975). The moral judgment of the child. London: Routledge and Kegan Paul. (Original work published 1932).

Ratcliff, R. (1993). Methods for dealing with reaction time outliers. Psychological Bulletin, 114, $510-532$.

Sabbagh, C., Dar, Y., \& Resh, N. (1994). The structure of social justice judgments: A facet approach. Social Psychology Quarterly, 57, 244-261. 
Schmitt, M. (1996). Individual differences in sensitivity to befallen injustice. Personality and Individual Differences, 21, 3-20.

Schmitt, M., Gollwitzer, M., Maes, J., \& Arbach, D. (2005). Justice sensitivity: Assessment and location in the personality space. European Journal of Psychological Assessment, 21, 202-211.

Smith, E. R. (1994). Procedural knowledge and processing strategies in social cognition. In R. S. Wyer Jr. \& T. K. Srull (Eds.), Handbook of social cognition: Vol. 1, Basic processes (2nd ed., pp. 99-151). Hillsdale, NJ: Erlbaum.

Smith, E. R., \& Lerner, M. (1986). Development of automatism of social judgments. Journal of Personality and Social Psychology, 50, 246-259.

Strack, F., \& Deutsch, R. (2004). Reflective and impulsive determinants of social behavior. Personality and Social Psychology Review, 8, 247-320.

Todorov, A., \& Uleman, J. S. (2002). Spontaneous trait inferences are bound to actors: Evidence from false recognition. Journal of Personality and Social Psychology, 83, 1051-1065.

Turiel, E. (1983). The development of social knowledge: Morality and convention. Cambridge: Cambridge University Press.

Uleman, J. S. (1999). Spontaneous versus intentional inferences in impression formation. In S. Chaiken \& Y. Trope (Eds.), Dual-process theories in social psychology (pp. 141-160). New York: Guilford press.

Uleman, J. S., Adil Saribay, S., \& Gonzalez, C. M. (2008). Spontaneous inferences, implicit impressions and implicit theories. Annual Review of Psychology, 59, 329-360.

Uleman, J. S., Hon, A., Roman, R., \& Moskowitz, G. B. (1996a). On-line evidence for spontaneous trait inferences at encoding. Personality and Social Psychology Bulletin, 22, 377-394.

Uleman, J. S., Newman, L. S., \& Moskowitz, G. B. (1996b). People as flexible interpreters: Evidence and issues from spontaneous trait inference. In M. P. Zanna (Ed.), Advances in experimental social psychology (Vol. 28, pp. 211-279). San Diego, CA: Academic Press.

Van den Bos, K., Ham, J., Lind, E. A., Simonis, M., Van Essen, W. J., \& Rijpkema, M. (2008). Justice and the human alarm system: The impact of exclamation points and flashing lights on the justice judgment process. Journal of Experimental Social Psychology, 44, 201-219.

Van den Bos, K., Peters, S. L., Bobocel, D. R., \& Ybema, J. F. (2006). On preferences and doing the right thing: Satisfaction with advantageous inequity when cognitive processing is limited. Journal of Experimental Social Psychology, 42, 273-289. 